\title{
Editoriali
}

\section{Epidemiologia e Psichiatria Sociale (EPS) per una utilizzazione più estesa dell'approccio epidemiologico e del metodo scientifico nella psichiatria sociale italiana}

\author{
MICHELE TANSELLA
}

\section{LE CIRCOSTANZE FAVOREVOLI, LE SITUAZIONI PREDISPONENTI, GLI EVENTI PRECIPITANTI}

La decisione di fondare una nuova rivista scientifica non è mai legata ad un unico fattore «causale», ma rifleite piuttosto l'esistenza di una serie di circostanze favorevoli e di situazioni predisponenti. Tali circostanze e tali situazioni, tuttavia, spesso produrrebbero solo intenzioni e progetti, se non intervenissero anche eventi precipitanti, in grado di trasformare le intenzioni in decisioni ed iniziative concrete.

Prima di analizzare le variabili che hanno determinato, in modo tipicamente multifattoriale, la nascita di EPS, è opportuno menzionare quella che a me sembra la componente principale del processo di fondazione di questa rivista, vale a dire l'aver preso atto della crescente importanza, nel nostro paese, della psichiatria epidemiologica e dell'approccio epidemiologico in psichiatria.

Il peso di tale disciplina, o se si vuole di tale approccio, che altrove ha storia e tradizioni solide ed antiche e che per noi rappresenta una relativa novità (Tansella, 1985), è rapidamente aumentato negli ultimi anni anche in Italia. Come ha scritto John Ziman (1976) «the hallmark of a new discipline is the establishment of a specialized journal catering to the scholarly needs of its exponents. It constitutes an act of solidarity and sodality, and polarizes the subject around it». Dunque è sembrato giunto il momento che coloro che sono interessati alla utilizzazione del-

Indirizzo per la corrispondenza: Professor M. Tansella, Servizio di Psicologia Medica, Istituto di Psichiatria, Ospedale Policlinico, 37134 Verona.

Fax $(+39) \quad 045-585.871$ l'approccio epidemiologico in psichiatria, nella pratica clinica come nella ricerca, avessero un comune punto di riferimento, una loro rivista. Da essi, dalla loro capacità di fornire stimoli culturali e di dare un contributo attivo per sviluppare questo punto di riferimento comune, dipenderà il futuro di questa rivista.

Detto questo occorre ricordare le circostanze favorevoli, le situazioni predisponenti e gli eventi precipitanti a cui ho accennato prima. Tra le circostanze favorevoli occorre menzionare il cambiamento del «clima culturale», cui si riferisce De Salvia nel suo articolo, pubblicato in questo fascicolo di EPS (De Salvia, 1992); vale a dire il passaggio, nella psichiatria italiana più avanzata e più attenta a mantenere elevato il livello della riflessione critica sulle teorie e sulla pratica della nostra disciplina, dalla impostazione sociopolitica fortemente ideologizzata degli anni ' 60 e ' 70 a quella sociopsichiatrica ed epidemiologica degli anni '80. Tra le situazioni predisponenti citerei innanzitutto la mancanza di una rivista italiana del tipo di EPS, cioè una rivista in grado di raccogliere quei contributi che sono attualmente dispersi in almeno 5 o 6 riviste diverse, nessuna delle quali specializzata nel settore dell'epidemiologia e della psichiatria sociale. Ma è necessario ricordare anche la disponibilità e l'interesse mostrato dall'Editore, Il Pensiero Scientifico di Roma, che ha deciso di investire e di impegnare la sua esperienza (e non soltanto quella) in questa nuova, non facile, impresa editoriale.

L'evento precipitante più importante è stato infine la fondazione della Società Italiana di Epidemiologia Psichiatrica (SIEP) avvenuta nell'aprile 1989. L'interesse che tale nuova società scientifica ha destato ha rappresentato un segno che mi è sembrato opportuno leggere e tradurre in una nuova iniziativa culturale, la nascita di EPS. Tale rivista, pur non essendo legata alla Società, dalla quale anzi è del tut- 
to indipendente e autonoma, vuole costituire anche un mezzo di diffusione delle iniziative scientifiche e culturali della SIEP e dei soci della SIEP.

\section{IL COLORE, GLI OBIETTIVI, IL TITOLO}

Michael Shepherd (1986), ricordando la nascita di Psychological Medicine, da lui fondata nel 1970, ha sintetizzato così «lo spirito» di quella fondazione: "Our initial task was to takle 3 questions, namely the colour of the dust-jacket, an agreement on objectives, and a title». Il primo problema fu da lui risolto scegliendo per la copertina della nuova rivista il colore grigio (in quanto nulla in psichiatria è bianco o nero); il secondo decidendo di identificare come obiettivi della rivista la pubblicazione di lavori originali e di ottima qualità "across the wide spectrum of both psychiatry and its allied disciplines»; il terzo, il titolo, facendo "risorgere» il titolo della prima rivista inglese dedicata esclusivamente ai problemi delle malattie mentali, fondata da Winslow nel 1848 e da lui chiamata «Journal of Psychological Medicine and Mental Pathology" (Winslow, 1848).

Per quanto riguarda EPS voglio seguire il mio Maestro inglese almeno nella scelta dei problemi da affrontare se non nella loro soluzione. Per il primo ed il terzo non ci sono molte parole da spendere; la copertina è di colore verde perché è il colore della speranza, la speranza che questa nuova rivista possa dare un contributo non secondario per portare gli standard della psichiatria epidemiologica italiana a livelli europei; il titolo è stato scelto per sintetizzare la necessità di coniugare epidemiologia e psichiatria sociale, perché la seconda tragga dalla prima le conoscenze, le nozioni e la metodologia necessarie per essere «scientificamente fondata». Per quanto concerne il secondo problema, cioè la scelta degli obiettivi, è necessaria invece qualche parola in più. Farò riferimento questa volta a quanto detto da Alexander Leighton ad un recente Simposio della Section of Epidemiology and Community Psychiatry della WPA, per sintetizzare le componenti del «scientific cycle» (Leighton, 1991). Tali componenti, egli dice, possono essere così enumerate:

«1. scelta del problema sul quale investigare; 2 . formulazione del problema in termini che rendano possibile la ricerca, vale a dire: identificazione di una teoria pertinente, descrizione dei fenomeni pertinen- ti, dissezione del problema in una serie di ipotesi testabili mediante esperimenti e/o mediante osservazioni naturali; 3. sviluppo di tecniche in grado di testare le ipotesi (con eventuale controllo delle metodologie, revisione estesa delle ipotesi e riformulazione dei concetti e delle teorie che sottendono le ipotesi); 4. esecuzione della ricerca e raccolta dei risultati; 5. analisi ed interpretazioni dei risultati. Molti nuovi problemi sorgono a questo punto. Alcuni riguardano la riproduzione, la verifica e la revisione dei metodi, altri la riformulazione delle teorie e delle ipotesi e la produzione di nuove ipotesi. Il processo è quindi pronto per un nuovo ciclo e per molti altri cicli paralleli che possono procedere per linee diverse ma collegate».

Obiettivo di EPS è di incoraggiare una utilizzazione più estesa e diffusa, nella psichiatria sociale italiana, dei percorsi caratteristici del «ciclo scientifico». $\mathrm{Si}$ tenterà di raggiungere tale obiettivo anche attraverso la regolare collaborazione di referees per un lavoro di lettura critica dei contributi inviati per la pubblicazione. Ogni fascicolo conterrà inoltre Editoriali, scritti su invito, sia di autori italiani che di autori stranieri (questi ultimi in lingua inglese), che avranno lo scopo di fare il punto, in modo sintetico, su argomenti di attualità o su temi controversi. Infine alcune pagine della rivista potranno essere dedicate, come si è detto, alla pubblicazione di news relative alle attività della SIEP, oppure di circolari o di delibere di Regioni o ULSS su aspetti legislativi e normativi di interesse per gli psichiatri, per gli amministratori e per i tecnici ed i funzionari degli Enti locali e delle Regioni che si occupano di psichiatria.

\section{BIBLIOGRAFIA}

De Salvia D. (1991). I servizi postmanicomiali nella psichiatria sociale italiana. Il circolo pratica-ricerca. Epidemiologia e Psichiatria Sociale 1, 19-23.

Leighton A. (1991). Social science and psychiatry: a difficult relationship. Paper read at the Symposium on Psychiatric Epidemiology and Social Sciences (Oslo, 14-16 June 1991).

Shepherd M. (1986). Psychological medicine redivivus: concept and communication. Journal of the Royal Society of Medicine 79, 636-645.

Tansella M. (a cura di) (1985). L'Approccio Epidemiologico in Psichiatria. Boringhieri: Torino.

Winslow F. B. (1848). Preface. Journal of Psychological Medicine $1,3$.

Ziman J. M. (1976). Public Knowledge. Cambridge University Press: Cambridge. 